\title{
Eptifibatide Bolus Dose During Elective Percutaneous Coronary Intervention
}

\author{
Hossein Doustkami ${ }^{a}$, Saeed Sadeghieh Ahari ${ }^{b}$, Effat Irani Jam ${ }^{\text {c, d, }}$, \\ Afshin Habibzadeh ${ }^{\mathrm{a}}$
}

\begin{abstract}
Background: Eptifibatide is a platelet glycoprotein IIb/IIIa receptor antagonist used for the prevention of cardiac ischemic complications of percutaneous coronary intervention (PCI). Eptifibatide has been used with bolus dose only or bolus plus infusion in patients undergoing PCI which have shown less complications, but the risk of bleeding has been increased. We aimed to compare the outcome and bleeding rate of bolus dose alone or plus infusion in elective PCI.
\end{abstract}

Methods: In this quasi-experimental study, we compared the outcome of elective PCI following single bolus dose intracoronary (41 patients) or bolus plus intravenous infusion (19 patients) of eptifibatide. In-hospital and follow-up major adverse cardiac events (MACEs) and bleeding rate were recorded and evaluated between groups.

Results: Both groups were comparable regarding baseline findings. Bolus only compared to bolus plus infusion group had lower in-hospital (19.5\% vs. $31.6 \%)$ and follow-up MACE (15.4\% vs. $17.6 \%)$, lower bleeding in-hospital (14.6\% vs. $21.1 \%)$ and follow-up $(2.4 \%$ vs. $5.3 \%$ ) as well as lower mortality rate in hospital (4.9\% vs. $15.8 \%$ ), but higher follow-up mortality $(10.3 \%$ vs. 0$)$, but the difference was not significant.

Conclusions: We observed no significant difference regarding bleeding or MACE between intracoronary bolus infusion and bolus plus intravenous infusion of eptifibatide. It seems intracoronary bolus infusion of eptifibatide due to use of lower doses is a better choice in elective PCI to prevent post-PCI MACE.

Keywords: Eptifibatide; Bolus dose; Percutaneous coronary intervention

\footnotetext{
Manuscript submitted December 30, 2017, accepted January 29, 2018

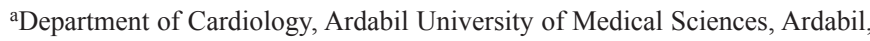
Iran

${ }^{b}$ Department of Social Medicine, Ardabil University of Medical Sciences, Ardabil, Iran

cDepartment of Internal Medicine, Ardabil University of Medical Sciences, Ardabil, Iran

${ }^{\mathrm{d} C o r r e s p o n d i n g ~ A u t h o r: ~ E f f a t ~ I r a n i ~ J a m, ~ I m a m ~ K h o m e i n i ~ H o s p i t a l, ~ A r d a b i l ~}$ University of Medical Sciences, Ardabil, Iran. Email: dr.jam733@gmail.com
}

doi: https://doi.org/10.14740/cr675w

\section{Introduction}

Coronary artery disease (CAD) is a major health problem worldwide [1]. Percutaneous coronary intervention (PCI) is a common revascularization strategy in the management of CAD due to its safety profile and ability to relieve symptoms and improve survival with low complications $[2,3]$.

However, stenting causes platelet activation and aggregation that can lead to catastrophic thrombotic complications. Various antiplatelet agents that block different pathways in platelet activation and aggregation, have been introduced and used for preventing cardiovascular events after coronary stenting [4]. Eptifibatide is a platelet glycoprotein $\mathrm{IIb} / \mathrm{IIIa}(\mathrm{GP} \mathrm{IIb} /$ IIIa) receptor antagonist that inhibits fibrinogen binding to the activated GP IIb/IIIa site which prevents platelet-platelet interaction and clot formation [5].

Previously, eptifibatide was mainly used with bolus dose following infusion which showed significantly less complication 30 days after intervention [4, 6-8], but it accompanies with the increased risk of bleeding [4]. Other studies have shown that single bolus dose only can be as effective as infusion by improving long-term survival [9-11].

Compared to other GP IIb/IIIa receptor antagonists and newly developed antiplatelet agents, eptifibatide (Integrilin) is often less expensive and more widely available in many hospitals $[12,13]$. In this study, we evaluated the in-hospital and 3-month follow-up outcome and bleeding rate of using single bolus or bolus plus infusion of eptifibatide in patients undergoing elective PCI.

\section{Materials and Methods}

A quasi-experimental study was performed on the patients confined for PCI elective in Ardabil Imam Khomeini hospital in order to investigate the therapeutic effect of eptifibatide in bolus dose compared to bolus plus maintenance. But the high prevalence of bleeding in the routine way which is not performed and the issues related to medical ethics did not allow designing a randomized controlled clinical trial study. So in a quasi-experimental trial study with all limitations, the results of treatment administering bolus dose of eptifibatide inside the coronary without infusion and the results obtained from the previous routine were compared. Inclusion criteria were patients who suffered cardiac ischemia, unstable angina, dan- 
Table 1. Baseline and Angiographic Findings Between Bolus and Bolus + Infusion Group

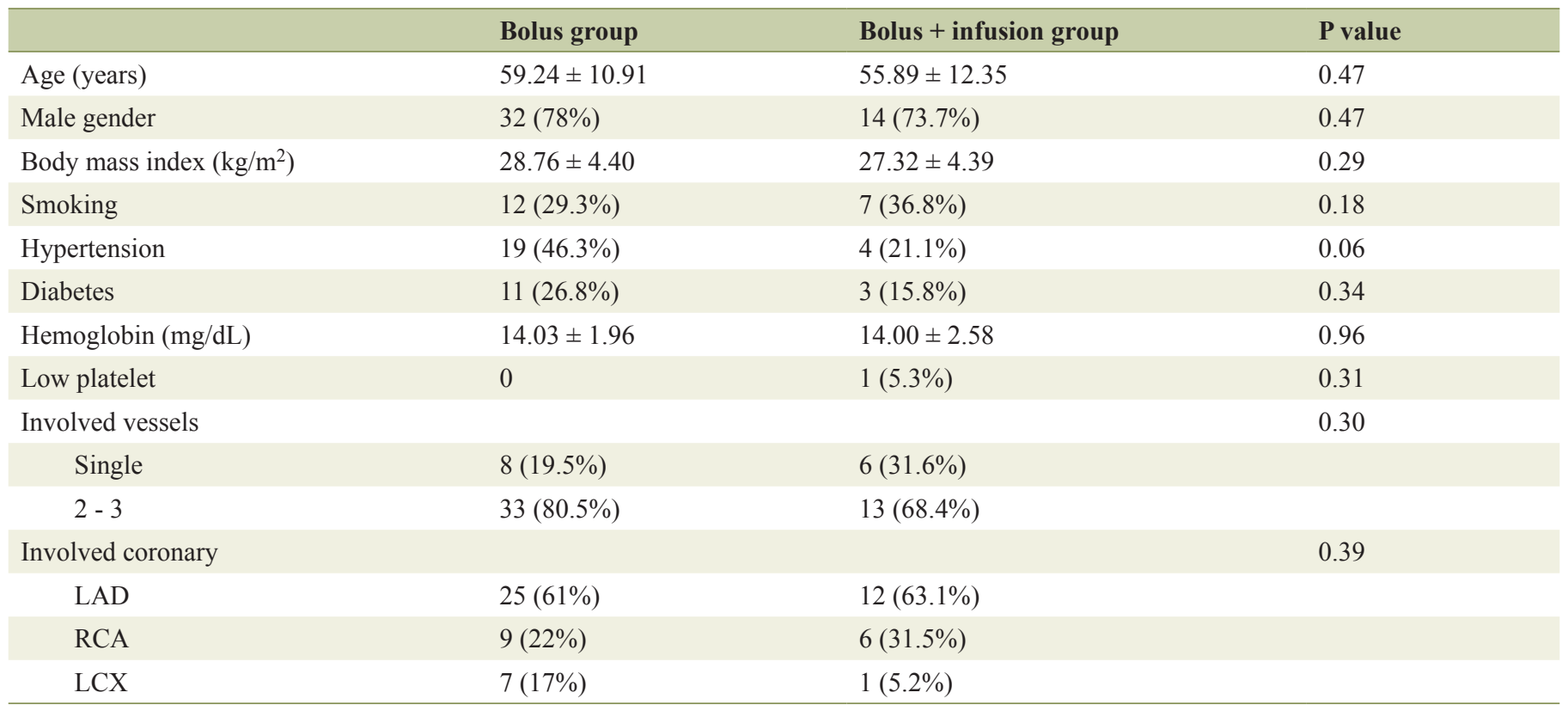

gerous coronary syndrome or big thrombus in coronary canal who underwent elective PCI. Patients with acute myocardial infarction (MI) in the first $24 \mathrm{~h}$, undergoing primary or rescue PCI, and recent PCI in the last 90 days were excluded. The Ethics Committee of Ardabil University of Medical Sciences approved the study protocol and written informed consents were taken from all participants.

Forty-one patients were included in the bolus group. The medical records of 19 eligible patients who received bolus dose of intracoronary with infusion were also reviewed as control group. Both groups were matched for age and sex. Data regarding baseline variables, including age, sex, body mass index (BMI), medical history, laboratory findings and angiography findings were recorded. MACEs such as death, MI, ischemia, and need for repeating revascularization and bleeding were evaluated during hospital stay and all patents were followed for 3 months.

\section{Statistical analysis}

All data were analyzed using SPSS17 (version 17; SPSS Inc., Chicago, IL). Results are expressed as mean \pm standard deviation or percentage. Clinical characteristics of two groups were compared using the Pearson Chi-square test for categorical variables and Student's $t$-test for continuous variables. P values of less than 0.05 were considered statistically significant.

\section{Results}

Baseline and angiographic findings of patients are demonstrated in Table 1. Both groups were comparable regarding baseline findings. Most cases in both groups were 2 - 3 vessels disease with LAD as the most common involved lesion.

In-hospital and during follow-up MACE are shown in Table 2. Although in hospital and during follow-up total MACE was higher in bolus + infusion group and higher mortality rate during follow-up in bolus group, there was no significant difference between groups. Bleeding rate was lower in bolus group.

\section{Discussion}

We compared the outcome and bolus only versus bolus plus infusion of eptifibatide in patients undergoing elective PCI and observed lower in-hospital and follow-up MACE and bleeding rate in bolus group, but the difference between groups was not significant.

Currently eptifibatide is recommended to be infused for 18 - $24 \mathrm{~h}$ after PCI; however, some studies have shown better outcome for shorter duration of drug infusion [14-17]. Fung et al [14] in the BRIEF-PCI trial, used a single bolus dose plus 2-h infusion of eptifibatide in low-risk patients undergoing PCI and found similar incidence of in-hospital MACE with no significant difference, but accompanied with significantly lower major bleeding, indicative of no need for prolonged infusion of eptifibatide, especially in low-risk patients. In another large study in 21,296 patients treated with eptifibatide, Gurm et al [15] showed that patients receiving eptifibatide bolus only had significantly lower rates of bleeding events and blood transfusion with no difference in adverse events.

Results of ESPRIT substudy [16] also noted that limited eptifibatide infusion in elective PCI could reduce bleeding risk and prevent ischemic incidence. Hess et al [17] also observed no increase in ischemic events, but reduced bleeding rate with shorter duration of eptifibatide infusion. 
Table 2. In-Hospital and During Follow-Up MACE Between Bolus and Bolus + Infusion Group

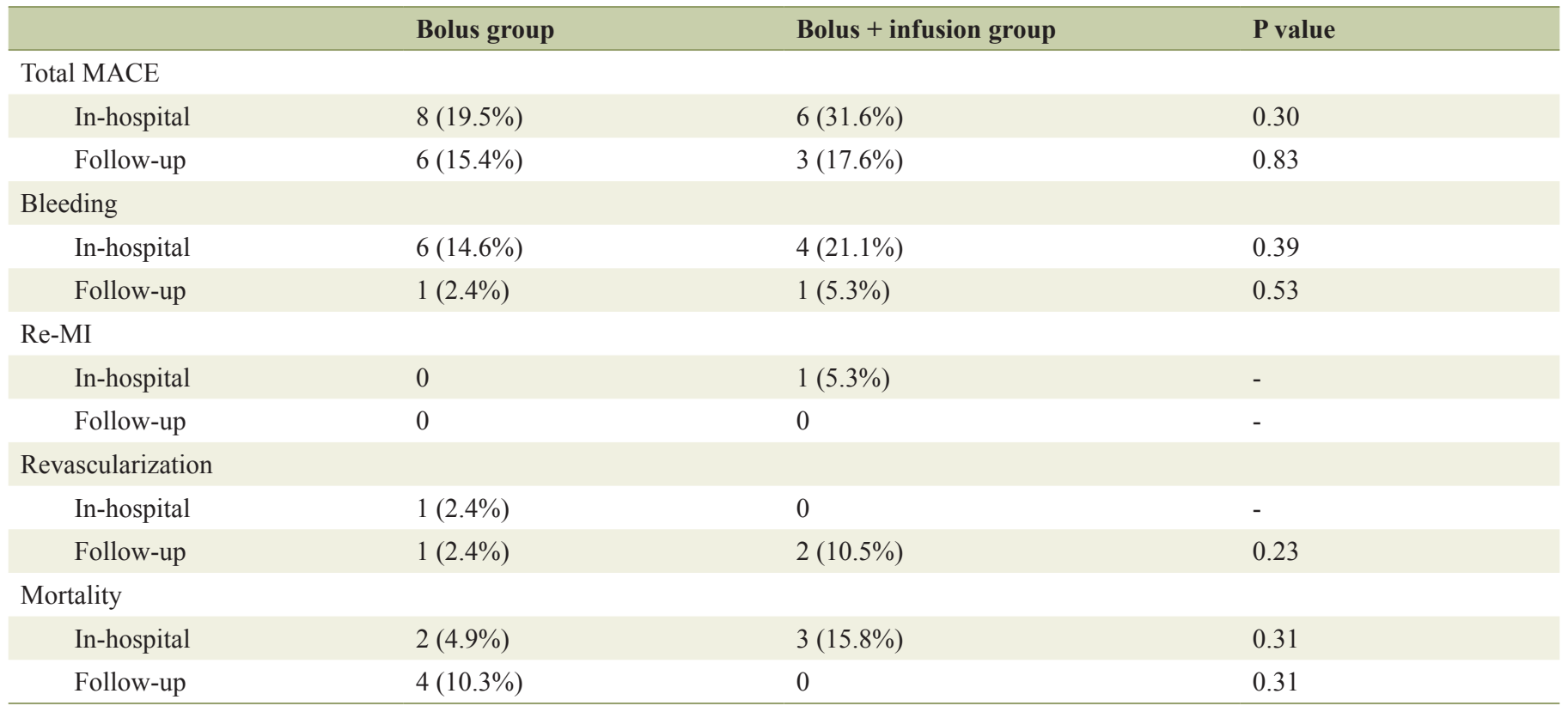

Similar results are reported for other GP IIb/IIIa antagonist. Kini et al [18] observed that bolus only versus standard dosing had similar outcome with reduced bleeding.

Hassan et al [19] have also reported that intracoronary infusion of eptifibatide seems to be safer and superior to intravenous and infusion mode with lower bleeding and complications.

Although most studies have reported that bolus only or with short duration has similar outcome with lower rate of bleeding, our study did not show significant difference in MACE or bleeding rate between groups, although the rate was lower in bolus only group. However, Marian et al [20] evaluated the efficacy of ticagrelor and eptifibatide bolus versus ticagrelor and eptifibatide bolus with 2-h infusion in high-risk acute coronary syndrome (ACS) patients undergoing early PCI and observed that the maximum platelet aggregation inhibition was achieved at $2 \mathrm{~h}$, with no significant bleeding indicative of the need for eptifibatide 2-h infusion. Regarding the literature, there is still need for further study to evaluate the exact dosage and protocol of eptifibatide infusion in patients undergoing elective PCI.

\section{Conclusion}

Based on analysis of the results obtained in present study, total MACE and bleeding in bolus dose were not significantly different from bolus + infusion of eptifibatide. It seems intracoronary bolus infusion of eptifibatide due to use of lower doses is a better choice in elective PCI to prevent post-PCI MACE.

\section{Acknowledgments}

This article has is extracted from the residency thesis from Ar- dabil University of Medical Sciences, Iran.

\section{Conflict of Interest}

The authors declare that they have no conflict of interest.

\section{References}

1. Mozaffarian D, Benjamin EJ, Go AS, Arnett DK, Blaha MJ, Cushman M, de Ferranti S, et al. Heart disease and stroke statistics - 2015 update: a report from the American Heart Association. Circulation. 2015;131(4):e29-322.

2. Patel MR, Dehmer GJ, Hirshfeld JW, Smith PK, Spertus JA. ACCF/SCAI/STS/AATS/AHA/ASNC 2009 appropriateness criteria for coronary revascularization: a report of the American College of Cardiology Foundation Appropriateness Criteria Task Force, Society for Cardiovascular Angiography and Interventions, Society of Thoracic Surgeons, American Association for Thoracic Surgery, American Heart Association, and the American Society of Nuclear Cardiology: Endorsed by the American Society of Echocardiography, the Heart Failure Society of America, and the Society of Cardiovascular Computed Tomography. Circulation. 2009;119(9):1330-1352.

3. Fihn SD, Blankenship JC, Alexander KP, Bittl JA, Byrne JG, Fletcher BJ, Fonarow GC, et al. 2014 ACC/AHA/ AATS/PCNA/SCAI/STS focused update of the guideline for the diagnosis and management of patients with stable ischemic heart disease: a report of the American College of Cardiology/American Heart Association Task Force on Practice Guidelines, and the American Association for Thoracic Surgery, Preventive Cardiovascular Nurses As- 
sociation, Society for Cardiovascular Angiography and Interventions, and Society of Thoracic Surgeons. J Am Coll Cardiol. 2014;64(18):1929-1949.

4. Pasala T, Sattayaprasert P, Bhat PK, Athappan G, Gandhi S. Clinical and economic studies of eptifibatide in coronary stenting. Ther Clin Risk Manag. 2014;10:603-614.

5. Boettcher BT, Olund TJ, Pagel PS. Acute severe thrombocytopenia occurring after administration of eptifibatide postpones emergent coronary artery surgery. Anesth Pain Med. 2016;6(4):e37575.

6. Therapy EIESotPIIRwI. Novel dosing regimen of eptifibatide in planned coronary stent implantation (ESPRIT): a randomised, placebo-controlled trial. Lancet. 2000;356(9247):2037-2044.

7. Platelet Glycoprotein IIiUARSUITTI. Inhibition of platelet glycoprotein IIb/IIIa with eptifibatide in patients with acute coronary syndromes. N Engl J Med. 1998;339(7):436-443.

8. Kleiman NS, Lincoff AM, Flaker GC, Pieper KS, Wilcox RG, Berdan LG, Lorenz TJ, et al. Early percutaneous coronary intervention, platelet inhibition with eptifibatide, and clinical outcomes in patients with acute coronary syndromes. PURSUIT Investigators. Circulation. 2000;101(7):751-757.

9. Marmur JD, Poludasu S, Lazar J, Cavusoglu E. Long-term mortality after bolus-only administration of abciximab, eptifibatide, or tirofiban during percutaneous coronary intervention. Catheter Cardiovasc Interv. 2009;73(2):214221.

10. O'Shea JC, Hafley GE, Greenberg S, Hasselblad V, Lorenz TJ, Kitt MM, Strony J, et al. Platelet glycoprotein IIb/ IIIa integrin blockade with eptifibatide in coronary stent intervention: the ESPRIT trial: a randomized controlled trial. JAMA. 2001;285(19):2468-2473.

11. Shariati H, Sanei H, Pourmoghadas A, Salehizadeh L, Amirpour A. Immediate outcomes of eptifibatide therapy during intracoronary stent implantation. Adv Biomed Res. 2016;5:204.

12. Gurm HS, Smith DE, Collins JS, Share D, Riba A, Carter AJ, LaLonde T, et al. The relative safety and efficacy of abciximab and eptifibatide in patients undergoing primary percutaneous coronary intervention: insights from a large regional registry of contemporary percutaneous coronary intervention. J Am Coll Cardiol. 2008;51(5):529-535.

13. Horne BD, Knight S, May HT. Panoptic total cardiovascular risk prediction using all predictors versus optimized risk assessment using variable subsets. Future Cardiol. 2012;8(5):765-778.

14. Fung AY, Saw J, Starovoytov A, Densem C, Jokhi P, Walsh SJ, Fox RS, et al. Abbreviated infusion of eptifibatide after successful coronary intervention The BRIEFPCI (Brief Infusion of Eptifibatide Following Percutaneous Coronary Intervention) randomized trial. J Am Coll Cardiol. 2009;53(10):837-845.

15. Gurm HS, Hosman C, Bates ER, Share D, Hansen BB, Blue Cross Blue Shield of Michigan Cardiovascular C. Comparative effectiveness and safety of a catheterization laboratory-only eptifibatide dosing strategy in patients undergoing percutaneous coronary intervention. Circ Cardiovasc Interv. 2015;8(2):e01880.

16. Rebeiz AG, Dery JP, Tsiatis AA, O'Shea J C, Johnson BA, Hellkamp AS, Pieper KS, et al. Optimal duration of eptifibatide infusion in percutaneous coronary intervention (an ESPRIT substudy). Am J Cardiol. 2004;94(7):926-929.

17. Hess CN, Schulte PJ, Newby LK, Steg PG, Dalby AJ, Schweiger MJ, Lewis BS, et al. Duration of eptifibatide infusion after percutaneous coronary intervention and outcomes among high-risk patients with non-ST-segment elevation acute coronary syndrome: insights from EARLY ACS. Eur Heart J Acute Cardiovasc Care. 2013;2(3):246255.

18. Kini AS, Chen VH, Krishnan P, Lee P, Kim MC, Mares A, Suleman J, et al. Bolus-only versus bolus + infusion of glycoprotein IIb/IIIa inhibitors during percutaneous coronary intervention. Am Heart J. 2008;156(3):513-519.

19. Hassan W, Al-Sergani H, Al Buraiki J, Dunn B, Al Turki F, Akhras N, Elshaer F, et al. Immediate and intermediate results of intracoronary stand-alone bolus administration of eptifibatide during coronary intervention (ICE) study. Am Heart J. 2007;154(2):345-351.

20. Marian MJ, Alli O, Al Solaiman F, Brott BC, Sasse M, Leesar T, Prabhu SD, et al. Ticagrelor and eptifibatide bolus versus ticagrelor and eptifibatide bolus with 2-hour infusion in high-risk acute coronary syndromes patients undergoing early percutaneous coronary intervention. J Am Heart Assoc. 2017;6:e05562. 